\title{
Gedanken zur Entwicklung der Zürcher Geographie in den letzten 40 Jahren *
}

\section{Wie ich zur Geographie kam}

Meine Großeltern mütterlicherseits lebten als Weinbauern im unteren Tößtal, mein Vater wurde auf einem einsam gelegenen Bergbauernhof im Zürcher Oberland geboren. Dank seinen naturkundlichen Interessen wurde er für mich früh zum Geographielehrer. Von ihm erfuhr ich von der Witterungsabhängigkeit der Wein- und Heuernte, vom Wassermangel im Sommer auf den Molassehöhen und etwas für mein Leben ganz Bestimmendes:

Ich mag die 3. oder 4 . Klasse besucht haben. An einem Sonntag spazierten wir zwei durch die wohl schönste Periglaziallandschaft der Schweiz mit Dellen und Kastentälern. Es ist dies der Höhenrücken zwischen Stadel und dem Gottfried-Keller-Dorf Glattfelden. Diese Periglaziallandschaft ist südwärts abgeschlossen durch einen bewaldeten Moränenwall. «Diese Hügel hat einst ein Gletscher aufgestoßen», erklärte mein Vater, gestützt auf Professor Heim. Gegen diese Autorität wagte ich keinen Widerspruch, keine Zweifel anzumelden - aber ich sah und fand die Gletscher nicht.

1941 kam ich nach Schiers, dem Geburtsort Eduard Imhofs. Die Evangelische Lehranstalt wurde für fünf Jahre meine Heimat. Dort im Prättigau reifte mein Entschluß zum Geographiestudium. Einerseits wollte ich erfahren, ob Vater und Professor Heim mit ihren Gletschern recht behielten, wie der Frost die Reben und das Wetter die Arbeit der Bergbauern beeinflußt. Anderseits begann mich die alpine Kulturlandschaft zu faszinieren, das Resultat des «Zusammenspiels» von Mensch und Natur, das ich während meines obligatorischen Landdienstes bei einem Prättigauer Bergbauern zu ahnen begann.

Meinem Geographielehrer in Schiers, einem Schüler von Professor Wehrli, verdanke ich viel: In seinem Unterricht lernte man selbständig denken, vom Auswendiglernen allein hielt er wenig. Im Mai 1944 brachte er mir die erste Nummer von der «Plan», der Schweizerischen Zeitschrift für Landes-, Regional- und Ortsplanung mit einem Begleitwort von Bundesrat Kobelt:

«Der Bund stellt einen Gesamtplan zur Bekämpfung der Arbeitslosigkeit auf (BRB vom 29. Juli

*Vortrag gehalten anläßlich der Jubiläumsfeier der GEGZ am 30. September 1989.
1942). . . . Dabei ist den Grundsätzen der allgemeinen Landesplanung nach Möglichkeit Rechnung zu tragen.» Diese Nummer enthielt einen Beitrag von Heinrich Gutersohn. Es ist sein bleibendes Verdienst, der Geographie in der Raumplanung eine Aufgabe zugewiesen zu haben - eine Chance, wie mir damals schien -, die sich für Geographen auftat. All dies bewog mich, Geographie zu studieren. $\mathrm{Zu}$ meinem Leidwesen riet mir der akademische Berufsberater von Zürich davon ab. «Geographie? keine Chance» argumentierte er, «beispielsweise benötigen die Zürcher Kantonsschulen nur drei Geographielehrer (auf eine dieser drei Stellen wurde ich 1957 gewählt!), wovon willst Du sonst leben?» Dieser Ausspruch macht deutlich, zu was die Geographie damals gestempelt war: Ein Fach für die Schule!

Trotzdem: Durch meinen Vater ermuntert blieb ich dabei und verschrieb mich der Geographie. Im Rückblick auf die vergangenen 40 Jahre darf ich festhalten: Meine Motivation erwies sich als tragfähig. Die Arbeiten meiner Schüler zur Solifluktion, einer Frosterscheinung von Hochgebirgsböden, zur Gletschergeschichte und zur alpinen Kulturlandschaft sind Belege dafür.

\section{Wo wirken heute Geographen in unserer Gesellschaft, wovon leben sie?}

Wie würde mein damaliger Berufsberater heute urteilen? Was ist aus unseren, meinen Schülern geworden? Sind Geographen immer noch fast ausschließlich als Lehrer tätig? Oder nützen sie ihre Chance die Verflechtung Mensch-Natur -, zur Lösung von Problemen unserer Zeit beizutragen?

Als Lehrer waren vor acht Jahren noch die Hälfte unserer ehemaligen Hauptfachstudenten tätig, seither ist diese Zahl gesunken. An Universitäten und anderen Hochschulen wirken $1 / 7-1 / 8$ unserer Ehemaligen. Unter Berufen der «angewandten Geographie» seien Tätigkeiten verstanden, die mit unserem Fach in Beziehung stehen. Dazu zählen beispielsweise Tätigkeiten in der Raum- sowie in der Landschaftsplanung, Entwicklungshilfe, Wirtschaftsför-

Gerhard Furrer, Prof., Dr., Geographisches Institut der Universität Zürich-Irchel, Winterthurerstrasse 190, 8057 Zürich 
derung und -beratung, im Tourismus, Natur- und Umweltschutz. Diese Zahl steigt gegen $1 / 3$. Rund $1 / 5$ unserer Absolventen sind in Berufen ohne oder mit nur losem Bezug zur Geographie tätig. Ich denke an Mitarbeit in Banken und Versicherungen, Redaktionen, Statistischen Ämtern. Das Berufsfeld des Geographen hat sich in der Nachkriegszeit also verändert, der Trend führt meines Erachtens weiter von der Lehrtätigkeit weg.

In Zusammenhang mit drei Umfragen lieferten die Ehemaligen nebst Angaben zu ihrer beruflichen Tätigkeit auch Vorschläge, um die Chancen von Geographen auf dem Arbeitsmarkt zu erhöhen. So wird in den Antworten starkes Gewicht auf praxisbezogene Ausbildung gelegt. Diese sollte jedoch nicht nur in Form von Seminarien oder vergleichbaren Veranstaltungen in den Lehrgang der Geographie eingebaut werden, auch außerhalb der Universität seien praktische Erfahrungen zu sammeln. Dies würde dem einzelnen auch ermöglichen, seine Neigungen innerhalb der Geographie besser kennenzulernen.

Die weitgefächerte Allgemeinbildung des Geographen wird von den Ehemaligen begrüßt. Neben dem Hauptfach Geographie sollte ein vertieftes Studium eines andern Faches erfolgen. Dieser Forderung unserer Ehemaligen kommt das Diplomreglement der philosophischen Fakultät II entgegen, indem zu jedem Hauptfachstudium einige Nebenfächer zu absolvieren sind und im Schlußexamen vom Hauptfach abgesehen - ein «großes» Nebenfach geprüft wird. Im Hinblick auf eine breite Ausbildung sind für das Geographiestudium die Nebenfächer Mathematik und Geologie obligatorisch. Dank dem Verständnis, das die Fakultät dem Wesen und Bedürfnis der Geographie entgegenbringt, können Geographen auch Nebenfächer an der rechts- und staatswissenschaftlichen Fakultät sowie an der philosophischen Fakultät I belegen und mit einem Examen abschließen. Ohne besonderes Gesuch sind das die Fächer Volkswirtschaftslehre, Ethnologie, Geschichte, Soziologie und Volkskunde. Eine besondere Bedeutung kommt dem «großen» Nebenfach zu: Studenten der Physischen Geographie wählen beispielsweise Geologie, jene der Humangeographie Volkswirtschaftslehre oder ein Fach der philosophischen Fakultät I. Diese Wahl bestimmt weitgehend die Spezialisierung innerhalb der Geographie. Im weiteren haben die jüngsten Umfragen gezeigt, daß eine gute EDV-Ausbildung für beinahe jede Berufsrichtung von sehr großem Nutzen ist.

\section{Der Ausbau des Geographischen Institutes der Universität}

Werden diese Wünsche, Forderungen von uns ernst genommen? Ein Blick in die Vorlesungsverzeich- nisse belegt das breite Angebot in EDV-Ausbildung, das wir mit der Berufung von Prof. Brassel erreichen konnten. Als neues Glied ist in der jüngsten Vergangenheit zur physischen und anthropogeographischen Seite unserer Wissenschaft die Quantitative Geographie hinzugekommen, insbesondere die geographische Informationsverarbeitung. Sie beschäftigt sich mit Methoden der Gewinnung, Darstellung und Analyse von Daten der Geosphäre. Bei der Datengewinnung kommt den Informationen, die Luftund Satellitenbilder zu geben vermögen, große Bedeutung zu: Denken wir an Länder der Dritten Welt, die über ungenügende statistische kartographische Grundlagen verfügen, Daten, welche für Entwicklungsprojekte wesentliche Voraussetzungen bilden.

Mit der Berufung des Kollegen Elsasser - er war lange Zeit am Institut für Orts-, Regional- und Landesplanung an der ETH tätig - wollten wir vermehrten Praxisbezug in der Ausbildung erreichen. Dazu gehören auch - vorläufig eher spärlich angeboteneLehrveranstaltungen von Praktikern, Ehemaligen im Berufsleben, zur angewandten Landschaftsplanung. Unsere Abteilung «Luft- und Satellitenbildinterpretation», deren Anfänge in die Mitte der vierziger Jahre zurückreichen, sowie die Abteilung «Kulturgeographie» sind aktiv an Entwicklungshilfeprojekten beteiligt und fördern damit den Einstieg unserer Studenten in diesen Bereich.

Die Nachkriegszeit geht als Ausbauphase der Zürcher Universitätsgeographie in die Geschichte ein: Von einem «Einmanninstitut» (Prof. Boesch) sind wir auf ein Institut mit sechs Lehrstühlen, einem breiten Mittelbau und zahlreichen Hilfskräften angewachsen. Damit ist auch ein «inneres» Wachstum verbunden: Das Lehrangebot ist breiter geworden, nicht zuletzt dank den beiden Professuren an der ETH. Neue kapital- und arbeitsintensive Methoden wie beispielsweise die Radiocarbondatierung, die Pollen- und Schwermetallanalyse wurden eingeführt, welche es uns erlauben, neue Probleme zu bearbeiten. Das Unterrichtsprogramm der quantitativen Geographie wird von beiden Geographieinstituten gemeinsam angeboten, Professoren von beiden teilen sich in die Lehre, die Computeranlage wird gemeinsam betrieben .

Unsere Hochschulgeographie hat leider auch Rückschläge erlitten: Durch den frühen Tod der Kollegen Müller und Bachmann sind angebahnte, vielversprechende Entwicklungen an beiden Instituten jäh abgebrochen worden. Ich denke an die Arktisforschung und an die Bearbeitung von Problemen der traditionellen Kulturlandschaft unserer Alpen.

Örtlich haben wir uns verlagert vom Hauptgebäude unserer Alma Mater über die Nagervilla an der Freiestraße zur Blümlisalpstraße. Im August 1983 zogen wir auf den Irchel, wo wir jetzt eine Bleibe haben. Dank dem Zürcher Volk durften wir bauen, unser «Heim» den gestiegenen Raumbedürfnissen - 
man denke an unsere vielfältigen Laboratorien - anpassen und mit dem Geographischen Institut der ETH unter dasselbe Dach ziehen.

In den letzten Jahren bin ich oft mit der Frage konfrontiert worden: Benötigt Zürich zwei geographische Hochschulinstitute? Es ist wohl nicht an uns, diese Frage zu entscheiden. Solange aber beide bestehen, wollen wir uns nicht konkurrenzieren, sondern in Lehre und Forschung uns gegenseitig ergänzen. Die Kollegen der ETH widmen sich besonders drei Bereichen, die bei uns an der Universität nicht als Schwerpunkte gepflegt werden. Es sind dies Themen der Klimatologie und der Hydrologie sowie die Humanökologie. Ein Geographiestudium in Zürich bietet daher besonders zahlreiche Vertiefungsrichtungen und außerdem Gelegenheit, bei besonders vielen Dozenten zu hören.

\section{Ein Wort zur Geographie an der Mittelschule}

Das Geographische Institut der Universität ist keine Familie mehr wie Ende der vierziger Jahre wir zählen seit Jahren über 300 Hauptfachstudenten; in den letzten zehn Jahren wurden 299 Diplome erteilt, 68 Studenten schlossen mit dem Doktorat ab. Weshalb dieser «Boom»? Soweit ich beurteilen kann, interessieren die Umwelt, der Lebensraum die jungen Menschen. Sie erleben als Gymnasiasten die Verwandlung und Bedrohung unserer Landschaft selber. Betroffenheit macht sich breit in der jungen Generation, besonders wenn sich ihr konkret die Überlebensfrage stellt.

Weil der Mensch beim aktuellen Umbruch (Beton in der Landschaft) als Mitverursacher verantwortlich ist und der junge Mensch spürt, daß auch er von seinem Lebensraum lebt und geprägt wird, interessiert ihn die Geographie - denn in ihr ist ja der Mensch selber eingeschlossen. Dieser echt verstandenen Geographie kommen unsere Mittelschulen durch starke Betonung der Humangeographie im Unterricht entgegen. Besonders wertvoll erwies sich der Einbezug von Gegenwarts- und Zukunftsfragen in den Geographieunterricht, in dem die Schüler bei vielen Kollegen am Gymnasium mit Umwelt- und Entwicklungsproblemen konfrontiert werden. Damit leisten unsere Gymnasiallehrer einen wertvollen Beitrag zur Bildungsaufgabe des Gymnasiums. Bei den künftigen Akademikern ist dadurch das Image des Schulfaches Geographie der Nachkriegsjahre verbessert worden. Einem unserer Kollegen der Mittelschule kommt dabei ein besonderes Verdienst zu: Emil Egli, der in der Öffentlichkeit weit über das Gymnasium hinaus die Anliegen der Geographie vertreten und das Ansehen unseres Faches durch Wort und Schrift gemehrt hat.

Studienanfänger führen gerne einen weiteren Grund für die Wahl der Geographie an, nämlich deren Breite, das weit gespannte Feld von Ausbildung und möglicher Betätigung. Daß sie dies erfahren haben, verdanken die jungen Studenten ihren Geographielehrern, deren zentrale Stellung für das Ansehen unseres Faches nicht genug hervorgehoben werden kann.

\section{Zur Stellung der modernen Geographie (Hans Boesch, 1962) - Geographie im Wandel unserer Zeit (Klaus Aerni, 1987)}

«Am Anfang der Geographie standen die Entdekkung neuer Erdteile sowie topographische Fragen, doch seither hat sich die Disziplin längst Problemen der Landschaft und der Umwelt ganz allgemein zugewandt» (AERNI, «NZZ»43/1987). «In dem Maße, wie sich die noch weiß verbliebenen Lücken auf unseren Erdkarten schlossen, wandte sich der Erdkundler der eingehenderen ... Untersuchung der Erdoberfläche zu.» Das Forschungsziel liegt heute «in der vertieften Erkenntnis und im besseren Verstehen des in großen Zügen schon Bekannten» (BOESCH, 1962).

Die Ära Boesch lieferte viele Beiträge zur Umschreibung unseres Untersuchungsobjektes. Hans Boesch schreibt: «Das Besondere des geographischen Untersuchungsobjekts liegt gerade darin, daß die verschiedensten Sphären sich in ihm zu einem Ganzen vereinigen, welches sowohl in seinen Teilen als auch in deren gegenseitigen Beziehungen zu studieren ist.» Dafür ist der Ausdruck "Landschaft» aufgekommen; besser schiene uns von der «Geosphäre» zu sprechen. Nicht wegzudenken in diesem Ringen um Grundsätzliches der Geographie sind Boeschs Schüler und Mitarbeiter Hans Carol und unser Kollege Ernst Winkler.

Ausgehend von dieser Auffassung der Geographie ergeben sich einige Probleme:

Das erste bildet die zunehmende Spezialisierung. Wir empfinden diese Entwicklung besonders, weil im geosphärischen Bereich viele verschiedenartige Dinge zusammenkommen. Unsere Forschungstätigkeit hat sich daher auf das Zusammenspiel der einzelnen Bausteine - Relief, Klima, Vegetation, Handlungen des Menschen, seine wirtschaftende Tätigkeit - zu konzentrieren. «Die heutige Situation kann vielleicht so charakterisiert werden, daß praktisch alle wissenschaftlich tätigen Geographen noch in der Lage sind, sich auf irgendeinem Teilgebiet als Spezialforscher zu betätigen (z. B. Geomorphologie). Die Zeiten einer wahrhaft umfassenden Beherrschung aller Sphären sind aber auf immer entschwunden.»

Ein zweites Problem liegt im dualistischen Charakter der Geographie: «Natur und Mensch tragen beide zur Gestaltung der Erdoberfläche bei, und beide sind als Faktoren bei der geographischen Untersuchung entsprechend zu würdigen.» Es kann festgestellt werden, daß der Geograph «in gleichem 
Maße den Erd- wie den Gesellschaftswissenschaften verpflichtet ist». «Unsere Hochschulen - und diese Feststellung gilt für die ganze Erde - gliedern dagegen die Wissensgebiete organisatorisch traditionell in Natur- und Geisteswissenschaften, wobei, wie etwa in Zürich, wichtige die Geographie direkt berührende Teilgebiete außerdem den Wirtschaftswissenschaften zugeordnet werden. An der Universität Zürich ist die Geographie der naturwissenschaftlichen Fakultät (philosophische Fakultät II) eingegliedert, ohne daß sich daraus irgendwelche Schwierigkeiten ergeben hätten, tragen doch in freizügiger Weise die Prüfungsbestimmungen und die verständnisvolle Haltung der verantwortlichen Kreise den besonderen Belangen der Geographie voll Rechnung.» - Meines Erachtens sind alle Bestrebungen, die Geographie auf zwei oder mehrere Fakultäten aufzuteilen, z. B. die Wirtschaftsgeographie der wirtschaftswissenschaftlichen, die Humangeographie der geisteswissenschaftlichen Fakultät zuzuweisen, abzulehnen. Das Beispiel der alpinen Kulturlandschaft mit ihren Erosions-, Abwanderungs- und Disparitätenproblemen zeigt sehr eindrücklich, daß sie selbst und ihre Probleme nur unter Beachtung natürlicher wie menschlicher Faktoren und deren Zusammenwirken verstanden und damit einer Lösung zugeführt werden können.

Als drittes Problem sei der unablässige Wandel der Landschaft erwähnt. Dieser langsame, oft nicht wahrnehmbare Prozeß kann im Sinne der Katastrophe durch plötzlich auftretende, tiefgreifende Umbrüche abgelöst werden. Wir denken an den Sommer 1987 mit seinen Gewitterfolgen oder seinen Bergstürzen, wobei deutlich wird, daß nicht nur natürliche Faktoren als Auslöser in Frage kommen können, hat doch der Mensch - etwa mit Rodungen oder beim Betrieb von Steinbrüchen - seine Hand mit im Spiel. Unser Untersuchungsobjekt ist also nicht statisch, es ist unter dem Gesichtspunkt des Wandels zu betrachten.

Unsere alpine Kulturlandschaft ist das Ergebnis einer jahrhundertelangen Entwicklung: Generationen von Menschen haben sie zur Fristung ihres Lebens geschaffen, indem sie ihre Wirtschaft und ihre Kultur unter meist optimaler Ausnützung der Natur gestalteten. Dieser Anpassungsproze $\beta$ des Menschen an das natürliche Angebot und die Umgestaltung der Natur durch die Bedürfnisse des Menschen geschahen wohl aufgrund generationenlanger Erfahrungen im Umgang mit dem Lebensraum. Die Kulturlandschaft ist daher ein komplexer Organismus: Die natürlichen und die anthropogenen Elemente, welche die Kulturlandschaft aufbauen, stehen untereinander in Beziehung und bilden in ihrer Gesamtheit das Landschaftsgefüge. Wenn daher auch nur ein einziges Landschaftselement ändert oder verändert wird, stellen sich immer gesamtlandschaftliche Folgen ein. Es gibt kein Einzelgeschehen in der Landschaft, welches sich nicht irgendwie auf das Land- schaftsganze auswirkt. "Das Postulat des Gesamtgefüges der Landschaft ist in der Geographie nach wie vor aktuell. Es bleibt jedoch das methodische Problem, auf welche Weise der Geograph als Generalist die landschaftlichen Funktionsbeziehungen über die Stufe der Beschreibung hinaus auf das Qualitäts- und Anspruchsniveau des Fachmannes aus der Spezialwissenschaft heben könne. Im Anspruch auf Integration verschiedener Teilbereiche im Rahmen eines ökologischen Systems liegt die Grundaufgabe der heutigen Geographie» (AERNI, «NZZ» 43/1987). Wenn der Mensch in das Landschaftsgefüge eingreift, Siedlungs- und Bewässerungsanlagen baut oder verfallen läßt, seine Bewirtschaftungsmethoden ändert, ist auf den gesamtlandschaftlichen Charakter Rücksicht zu nehmen. Damit sind wir bei der Umweltverträglichkeitsprüfung und der Gegenwart angelangt.

\section{Die Zukunft unseres Lebensraumes - eine Herausforderung für die Geographie}

Die Geographie ist Mitglied der Schweizerischen Akademie der Naturwissenschaften, der ehemaligen SNG. Im Rahmen der Akademie wirkt die geographische Kommission, in der alle unsere Hochschulinstitute, die Vereinigungen der Gymnasiallehrer und der berufstätigen Geographen sowie die Kartographie vertreten sind. Jährlich an drei Sitzungen in Bern werden unsere gemeinsamen Anliegen besprochen, wird «unsere Politik» festgelegt, werden Forschungsprojekte dargelegt, Symposien und die Öffentlichkeitsarbeit gefördert sowie Stellungnahmen zu Vernehmlassungen der Behörden formuliert. Man kennt sich, man zieht am selben Strick ich bin überzeugt, daß dieses Gremium viel Erfreuliches für die Stellung der Geographie in unserer Gesellschaft erreicht hat. So sind wir beispielsweise im Februar 1987 in der «NZZ» mit einem Aufsatz «Geographie im Wandel unserer Zeit», verfaßt von Klaus Aerni, an die Öffentlichkeit getreten. Seine Vorbereitung zwang die Mitglieder der Geographiekommission unsere Stellung, Aufgaben und Chancen grundsätzlich zu überdenken.

Die Bedeutung der Geographie im Rahmen unserer Gesellschaft dürfte in der nächsten Zukunft in den folgenden Bereichen liegen:

Palaeogeographie: In Zusammenhang mit der Gefahr einer möglichen Klimakatastrophe als Folge des Treibhauseffektes fördern die Regierungen zahlreicher Länder großangelegte Forschungsprogramme, liegt doch hier eines der zentralsten Probleme der Menschheit vor. Als Folge der Erwärmung ist mit Verschiebungen von Klimazonen zu rechnen, Dürren - besonders in heutigen Bewässerungsregionen am Rande der Ökumene - könnten Völkerwanderungen auslösen; als Folge der ther- 
misch bedingten Ausdehnung von Ozeanwasser und abschmelzendem Eis ist ein Meeresspiegelanstieg zu befürchten.

Diese Probleme sind nur interdisziplinär und international lösbar. Beispielsweise ist die Zusammenarbeit mit Physikern sinnvoll: Er versteht die im Eis und in Sedimenten gespeicherten «Botschaften» der Vergangenheit zu lesen. Botaniker, Pollenanalytiker andererseits zeigen uns frühere Vegetationszustände, sie benützen die Vegetation als Klimazeiger zur Klimarekonstruktion. Dem Wissenschafter stellt sich die bange Frage: Wann muß er im Laufe seines Erkenntnisprozesses Behörden und Öffentlichkeit warnen? Ich glaube, daß er sich schon äußern muß, bevor er mit absoluter Sicherheit eine auf uns zukommende Katastrophe erkennen kann sonst kommt seine Warnung zu spät.

Welches ist bei diesem Problem der Anteil der Geographie? Klimaveränderungen der Gegenwart können nur richtig beurteilt werden, wenn der Rahmen bekannt ist, indem sich diese in der Vergangenheit, unbeeinflußt vom Menschen, abgespielt haben. $\mathrm{Zu}$ stände vor und nach menschlichen Eingriffen interessieren daher besonders, so beispielsweise vor und nach großen Rodungsphasen seit der ausgehenden Jungsteinzeit oder vor und nach Einsetzen der Industrialisierung. Man sucht nach «Analogiefällen der Vergangenheit»: Klimaphasen mit höheren Temperaturen der Atmosphäre und deren Auswirkungen auf Mensch und Umwelt. Zeitreihen von Temperatur, Niederschlag und Albedo sowie Ausmaß und Geschwindigkeit von Klimaschwankungen und die Antwort der Ökosysteme auf solche Schwankungen in der Vergangenheit bilden eine Grundlage für die Modellbauer unter den Meteorologen, damit diese die Klimaentwicklung analysieren, erklären und verstehen können. Und außerdem: Die Modellbauer benötigen die Ergebnisse palaeoklimatischer Forschung, um ihre Modelle zu testen, fehlen ihnen doch lange, weit zurückreichende meteorologische Beobachtungsreihen.

Umweltproblematik: Folgen von Handlungen des Menschen auf den Landschaftshaushalt sind an konkreten Beispielen zu untersuchen. Ich denke etwa an Erosionsschäden in Kulturland infolge Übernutzung und falscher Bewirtschaftung, an die Freisetzung von $\mathrm{CO}_{2}$ infolge der Nutzung fossiler Brennstoffe, an die Folge von Luftverschmutzung auf unsere Böden. Beispielsweise studiert einer meiner Mitarbeiter mögliche Auswirkungen von Autotunnelentlüftungen, die Deponie von Schadstoffen aus der Luft in Vegetation und Boden. Die Umweltverträglichkeitsprüfung hat in den letzten Jahren endlich in unserer Gesellschaft die notwendige Beachtung gefunden. Bei dieser drängt sich die Mitarbeit der Geographen direkt auf. Aus ihrer Kenntnis des Landschaftsgefüges sind die Geographen prädestiniert, Beiträge zur Lösung von Problemen unserer
Zeit - besonders an der «Schnittstelle» Mensch Natur - zu liefern.

\section{Landesplanung und Entwicklung peripherer}

Räume: Unsere Mitarbeit in der Raumplanung geht - wie eingangs erwähnt - auf Heinrich Gutersohn zurück und hat heute ihren festen Stellenwert. Wesentlich ist dabei lediglich, daß die Geographen ihre Stellung in diesem Aufgabenbereich halten. In der Vermittlung geographischer Erkenntnisse zuhanden der Planung, der Entwicklung zurückgebliebener Regionen sowie in der Mitarbeit in der Entwicklungshilfe liegen unsere Aufgaben der nächsten $\mathrm{Zu}$ kunft. Aus der Landschaftsanalyse, aus dem Studium der Funktionszusammenhänge Raum Mensch - Zeit sollten Spielräume menschlichen Handelns aufgedeckt werden, welche ökologische Grenzen respektieren.

Die Schweiz und ihre räumliche Entwicklung werden durch den europäischen Integrationsproze $\beta$ stark betroffen - und zwar unabhängig von den Modalitäten der Annäherung an die EG. Untersuchungen über die Auswirkungen der wirtschaftlichen Integration Europas auf Raumordnung und Siedlungsstruktur der Schweiz fehlen weitgehend. Hier besteht eine wichtige Forschungslücke, welche durch geographische Arbeiten verringert werden sollte. Der Beitrag der Schweiz zu Europa könnte u. a. darin bestehen, im Sinne des erwachenden «Regionalismus» föderalistische Entwicklungen zu fördern. Dies wäre ein substantieller Beitrag für eine Akzentverschiebung der EG-Politik, die dringend notwendig erscheint, und wofür wachsendes Verständnis sichtbar wird (ELSASSER, «NZZ» 31, 1989).

Wie jeder Organismus unterliegt auch die Kulturlandschaft fortwährendem Wandel. Dieser erfolgte vielerorts in unserem Lande nicht im Sinne eines evolutionären Vorgangs, sondern als tiefgreifender Umbruch, ohne Rücksicht auf gesamtlandschaftliche Folgen. Um solche schädlichen Entwicklungen zu verstehen und zu verhindern, benötigen wir umfassend ausgebildete Geographen, die als Raumplaner in der Lage sind, notwendige Eingriffe mit dem Blick auf das geosphärische Gesamtgefüge anzuregen. Es geht dabei um die Frage: «Auf welche Weise verändert das Handeln des Menschen seine konkrete Umwelt und wie müßte sein Handeln aussehen, das nicht seine eigenen Voraussetzungen zerstört?» (BÄTZING, «MAB» 27, 1988).

\section{Ausblick}

In unserer Zeit, da Spitzenwissenschafter in verschiedenen Disziplinen, Paul Niggli und Carl Friedrich von Weizäcker 1948 als früheste, nach Synthese drängen und eine erneut ganzheitlich gerichtete 
Philosophie die Überlebensmöglichkeit nur in der «Geborgenheit im Raum» sieht, ist unsere geographische Wissenschaft deutlich angesprochen und konkret gefordert. Damit erhält die Landschaftskunde einmal mehr große Bedeutung: Es geht um die Synthese Natur - Mensch unter der räumlichen (landschaftlichen) Perspektive, um die Synthese von Landschaftselementen, um die Verbindung Natur-Mensch in ihren gegenseitigen Abhängigkeiten und Beziehungen, um das Landschaftsgefüge. Darin liegt unsere Chance!

Mein Kollege Karl Henking, Professor für Ethnologie, stellte mir die folgenden Gedanken zu meinem Vortragstext zur Verfügung, die ich - weil sie sehr beachtenswert sind - gerne weitergebe:

Der Begriff des Landschaftsgefüges muß das Leitmotiv einer in die Zukunft weisenden Geographie sein. Es geht dabei um eine Synthese, nämlich um das ganzheitliche, holistische Verstehen der gegenseitigen Beziehungen zwischen Mensch und Natur. Darin liegt keineswegs eine Rückkehr zu überlebter Naturromantik, auch nicht die Utopie eines bloßen Schuldbekenntnisses des Menschen über die Zerstörung der Natur. Vielmehr bedeutet das ganzheitliche Verstehen des Landschaftsgefüges ein sorgfältiges Überdenken der Stellung des Menschen in der Welt überhaupt und bringt so eine Daseinsphilosophie, die dem Geographen seine geistige Grundlage gibt, sein wissenschaftliches Forschen prägt und sein Handeln bestimmt. Denn am Grunde jeder Wissenschaft ruht die Philosophie, nämlich das Denken über Wesen und Sinn des Menschen, und es liegt auf der Hand, daß bei einem ganzheitlichen Verstehen des Landschaftsgefüges ein neues Denken über den Menschen nötig ist.

Das ist freilich nicht so leicht, denn das bisherige Bild vom Menschen in unserer abendländischen $\mathrm{Zi}$ vilisation gründete im wesentlichen auf drei Denktraditionen: Der jüdisch-christlichen, der antikgriechischen und der neuzeitlich-naturwissenschaftlichen.

Nach der jüdisch-christlichen Tradition gilt die Welt als Schöpfung Gottes. Darin wird dem Menschen eine Spitzenposition als das allen Dingen und Wesen übergeordnete Geschöpf zugeschrieben («Macht euch die Erde untertan»). Diese Position legt dem Menschen aber eine Verantwortlichkeit Gott gegenüber als Beschützer der Schöpfung auf («Ich anvertraue euch die Erde»). Beide, Welt und Mensch, sind durch den Willen Gottes als dessen bewußtes Handeln entstanden, als Teil eines universalen Planes Gottes. Die daraus abzuleitende Konsequenz für unser Thema, der Beziehung zwischen Mensch und Natur in einem ganzheitlichen Landschaftsgefüge, würde bedeuten, daß der Mensch sich dem Plane Gottes eingefügt erkennt und aus dieser Ein- und Unterordnung denkt, plant und handelt. Er kann also nicht nach eigenem Gutdünken entscheiden, sondern muß zuerst den Willen Gottes erkunden und erkennen.

In der antik-griechischen Tradition nimmt der Mensch eine Sonderstellung in der Welt ein, weil er mit Vernunft («logos») begabt ist und infolge dieser Eigenschaft das «Wie» und «Warum» aller Erscheinungen erfassen und beschreibend mit Wortbegriffen definieren kann. Auch in der den Menschen umfassenden Welt ist Vernunft wirksam, nun freilich eine übermenschliche, an der der Mensch als einziges von allen Wesen allerdings Anteil hat. Die daraus sich ergebende Konsequenz besteht darin, daß der Mensch Entscheidungen selbst zu fällen vermag, jedoch im Rahmen und aufgrund der Gesetze der Vernunft. Ihnen ist er eingeordnet, und durch sie sind sein Denken, Planen und Handeln bestimmt und auch begrenzt.

Nach der neuzeitlich-naturwissenschaftlichen Tradition ist der Mensch in der Entwicklungsgeschichte des Universums und des Planeten Erde ein Spätling und lediglich das Produkt einer Differenzierung aus tierischen Vorformen und von diesen im wesentlichen durch eine Überentwicklung des Gehirns unterschieden. Aber gerade diese Absonderlichkeit befähigt ihn, die Gesetze des Seins in der Welt analytisch zu erkennen. Die Konsequenz dieses analytisch-diachronen Denkens liegt darin, daß der Mensch sich selbst zum Schöpfer machen kann.

Diese drei Traditionen des Denkens über den Menschen folgen sich zwar in der Geschichte, überschneiden und vermischen sich aber auch, werden einseitig verstanden oder dogmatisiert und geraten in verwirrende Gegensätze. Aus ihnen entstand das heute weitgehend gültige Selbstverständnis des Menschen als eines Wesens, das Kraft seiner Erkenntnis und seines Wissens Macht über alle Dinge und über alle anderen Wesen besitzt und deshalb das Recht hat, alles Sein in der Welt nach seinem Willen und Begehr zu manipulieren.

Das Ergebnis dieser Hybris kennen wir alle: Raub an der Substanz des Lebens, Zerstörung der Natur, Gefährdung des Menschseins. Allenthalben erheben sich allerdings nun warnende Stimmen und Ratschläge zur Bannung des Unheils. Hier hat auch der Geograph als ein Wissender um das Landschaftsgefüge als einer Synthese zwischen Natur und Mensch die Pflicht, sich einzusetzen. Dabei geht es vor allem und wesentlich darum, eine neue Definition des Menschen zu entwickeln. Vielen mag das als ein rein theoretisch-philosophisches oder weltanschaulichreligiöses, allenfalls psychologisches Problem erscheinen. Es ist aber auch ein eminent geographisches, deshalb nämlich, weil der Geograph die Eigenschaften der Erde erforscht und durch die so gewonnene Einsicht in diese Eigenschaften erkennt, wie sie einerseits auf das Dasein des Menschen wirken und wie andererseits der Mensch mit ihnen umgeht. Aufgrund dieser Einsicht bieten sich dem 
Geographen Ansätze zu dem dringend benötigten neuen Denken über den Menschen.

Diese Ansätze sollen in drei Punkten kurz umschrieben werden:

1. Aufgrund der wissenschaftlichen Erkenntnis der inneren Zusammenhänge im Landschaftsgefüge als einer Synthese zwischen Natur und Mensch erweist sich der Mensch als Teil des Naturganzen. Er ist nicht der Beherrscher der Natur, sondern ein ihr eingeordnetes und damit im Letzten begrenztes Wesen.

2. Diese wissenschaftliche Erkenntnis hat ein ethisches Prinzip zur Folge: Der Mensch darf die Macht des Wissens, die er durch seine wissenschaftliche Erkenntnis besitzt, nicht zur Manipulierung des Landschaftsgefüges mißbrauchen. Seine ethische Aufgabe besteht darin, zu bewahren und zu pflegen, statt zu herrschen.

3. Aus dem ethischen Prinzip ergeben sich Richtlinien für das konkrete praktische Handeln des
Menschen. Sie heißen: Ehrfurcht, Bescheidenheit und Rücksicht.

Aus diesen Überlegungen ergibt sich für uns Geographen eindeutig die Verantwortung, die wir als Forscher und als Menschen erfüllen müssen im Lebensgefüge von Landschaft und Mensch.

\section{Literaturverzeichnis}

AERNI, K.: Geographie im Wandel unserer Zeit. "NZZ” 43, 1987.

BÄTZING, W.: Ökologische Labilität und Stabilität der alpinen Kulturlandschaft. "MAB-Information" 27, 1988.

BOESCH, H.: Zur Stellung der modernen Geographie. Geographica Helvetica 4, 1962.

ELSASSER, H.: EG-Binnenmarkt und Raumordnung der Schweiz. "NZZ" 31, 1989.

FURRER, G. und FUTO, A.: Unsere Ehemaligen im Beruf. Geographica Helvetica 3, 1985. 